\title{
Integrating Human Factor Decision Components into A DES Model
}

\author{
Maji I. Abubakar \\ School of Mechanical and Design Engineering \\ University of Portsmouth \\ Portsmouth PO1 3DJ, UK \\ maji.abubakar@myport.ac.uk
}

\author{
Qian Wang \\ School of Mechanical and Design Engineering \\ University of Portsmouth \\ Portsmouth PO1 3dj, UK \\ Qian.wang@port.ac.uk
}

\begin{abstract}
Discrete event simulation (DES) is usually used for design, analysis and evaluation of a manufacturing system. For human-centered manufacturing systems, which employ human workers as one of main sources for production, systems designers often ignore human effects in development and evaluation of human-centered manufacturing systems using DES methods. This is partially because of complexity in human behaviours and a lack of adequate functionalities of the existing DES tools that allow incorporation of human factors into considerations, which may have an impact on the system performance. These missing parameters of human performance in a manufacturing system need to be addressed ideally at the early design stage, and thus there is a desire for creating a methodology, which enables system designers incorporating human factors (parameters) into DES tools. This paper presents a framework in development of an integrated DES platform, which allows an input of the relevant human related parameters into a DES model providing a more comprehensive simulation output for decision-makings on system performance.
\end{abstract}

Keywords-discrete event simulation; manufacturing systems, human factors; learning curve

\section{INTRODUCTION}

Biomechanical models applied for providing an understanding of adverse human effects that may have negative implications on system performance [1]. But these models cannot provide a straightforward answer in terms of an overall impact on system performance of human centered manufacturing operations, which are measured in relation to product cycle time, throughput, utilization of individual human workers and so on [2].

Discrete event simulation (DES) is a modelling simulation technique, which is widely accepted as an aided tool for design and analysis of manufacturing systems. Previous studies showed that $46 \%$ of automotive manufacturers applied DES methods to support production planning [3, 4]. Key performance indicators, which are throughput, utilizations, space allocations, inventory levels and so on, can be investigated using the DES tools. In automotive assembly, for instance, human centred manufacturing operations are still predominant at many plants in which assembly performed by workers may account for up to $50 \%$ of the entire process [3]. Greasley \& Owen [5] provided a latest review in modelling human behavior using the DES relating to human performance. Nevertheless, current DES tools have its own inadequacies in assessing human centered manufacturing activities in established DES models. In the existing DES tools, operators, who are designated to specific tasks or stations, are actually treated the same functionalities as such as machines. Boenzi et al. [6] suggested that aging ought to be integrated into DES tools for human centered system evaluations. Lassila et al. [7] studied a human centered assembly system by investigating utilization of resources (human and machines) leading to an increase in average lead-time from low worker utilizations and machine downtimes. In their systematic study Kolus et al. [8] explore the rationale linking human performance and quality output, observing the impacts of products design, process design and workstation design on the overall system performance. Perez et al. [9] investigated muscular fatigue and recovery pattern integrating biomechanical model into DES. Digiesi, et al. [10] examined learning and tiredness phenomena incorporating a model of worker behavior into a self-tailored DES tool and concluded that worker behavior has a significant impact on buffer levels and throughputs. Mason et al. [11] observed an overestimation of assembly line performance due to human behavior. Wang et al. [2] investigated the system variation due to influence of walking workers whose performance vary individually through a training process.

This paper presents a study by establishing a human factor decision tool (namely AutoHmot), which is a userfriendly platform developed using the Java language. AutoHmot allows human parameters (such as age and experience) as input into a DES tool. The study shows it can be an effective solution in evaluating effects of human factors on human centred performance of a manufacturing system.

\section{HUMAN FACTORS FOR MANUFACTURING SYSTEMS DESIGN AND EVALUATION}

The following notations are used

$$
\begin{aligned}
& \text { - } \quad T_{n}: \text { Time to produce } n^{\text {th }} \text { unit } \\
& \text { - } \quad \mathrm{Q}: \text { Incompressible factor } \\
& \text { - } \quad N: n^{\text {th }} \text { unit }
\end{aligned}
$$


- $G$ : Number of times similar operation is performed by an assembly operator

- $\quad D:$ Maximum number of assembly units

- $k_{1}=$ Existing age in years

- $F_{r m}$ : Remaining capacity in percentage after 38 years old

- $\quad k_{2}$ : Full capacity (100\%) at 38 years old

- $\quad L_{r}$ : Loss rate in percentage

- $T_{\max }$ : Assigned time to assemble first unit

- $\varepsilon$ : Decline rate of worker performance

- $\omega$ : standard time to complete a task regardless of the cumulative number of units produced

- $\quad C$ : Learning index which determines the speed of learning occurring each time as the cumulative output increases, it is computed as $\log (R) / \log (2)$ where learning rate $(\mathrm{R})$ is measured in percentage $(0<\mathrm{R}<1)$, e.g., $85 \%$ of learning rate $\mathrm{R}$ implies a reduction of $15 \%$ in cost of direct man labour hour needed to complete a subsequent unit.

Experience can be defined as the knowledge or a skill to be gained through involvement of a specific task, event or subject. In a process of human centered assembly, working experience improved individual human performance through learning after repeatedly performing an assigned task until time spent on completion of this task stabilized. Performance improvement of an individual is often measured in terms of a reduction of job cycle time, which is the result of the learning process. Thus, the learning curve theory is expressed in equation (1) with the parameter $\mathrm{Q}(0 \leq \mathrm{Q} \leq$ 1 ), where if $Q=$ zero, it indicates a task that is manually performed; if $\mathrm{Q}=$ one, it indicates a task that is predominantly executed by machines [6].

\section{A. Input data and analysis for simulation}

To account for worker variation in simulation experiments, activity times are collected in a sample of observations, which is fitted into a statistical distribution with a goodness of fit test between observed data and fitted theoretical data. However, difficulty may exist in data collection due to paucity of data, either it is because the system does not exist or it is not possible to obtain the data. Therefore, the input data based on estimations may not be sufficiently reliable; also in many cases it is hard to estimate. Instead, a probability distribution based on theoretical examination is often considered [12]. In manual tasks, most studies suggested the gamma distribution as the pattern of modelling activity time $[9,13]$. However, these distributions do not provide a favourable output. A goodness of fit $X^{2}$ test is used to test actual worker time distributions against the log-norma, gamma and beta distributions [11].

\section{B. Age and Experience}

A study through a literature review indicated that effect of worker job experience and aging has the most significant impact on worker performance in terms of variations in task time and average production rate [14]. In the literature study, it also shows that individual performance may decline from the certain age due to the natural decline of physical and physiological functions in such as visual ability, musculoskeletal force, flexibility/motion capability, memory or concentration and thermoregulation. On the other hand, as worker's age increases, it expects that the accumulation of experiences may also increase which also offset the decline of human abilities [15].

\section{Worker experience}

Definition of job experience is an issue of debate among scholars, some literature consider job experience as a function of time, which can be determined as the number of years spent on a job, organisation or position. Others described job experience as frequency or times an individual perform a particular task [16]. For this study, we define worker experience based on the cumulative number of similar tasks previously performed by the worker. In addition, assumed in this study that age of an assembly operator starts from the age of 20 years old and this operator will reach her/his full capacity at 38 years old. Workers are classified into three catogries based on levels of their experience: inexperiece worker who is a novice, experienced worker who has produced more than 20 units and highly experienced worker who has produced more than 40 units. Based on this hypothesis, time to produce unit by inexperienced worker at full capacity gained using the learning theory suggested by Anzanello \& Fogliatto [17]:

$T_{n}=\left[\left(Q+(1-Q) N^{C}\right)\right] T_{\max }$

Standard time to complete a task as required expressed as:

$\omega=T_{\max } \times Q$

Time to produce $n^{\text {th }}$ unit by experienced worker at full capacity taken as:

$T_{n}=\left[\left(Q+(1-Q) G^{C}\right)\right] T_{\max }$

\section{Aging}

Aging leads to human physiological decline that has undisputable effects on individual performance [6]. human physical capacity (to correlate with their performance) start to deteriorate between 35 to 40 years old, this phenomena was observed by many studies 
through a literature review. in this study, assuming a worker at 38 years old will reach his/her full human capacity, thus human capacity decline is obtained using equation $(4)[18,19]$ :

$$
L_{r}=0.57+0.012 k_{1}
$$

Thus, the remaining percentage of human capacity after a decline is determined by:

$$
F_{r m}=k_{2}-L_{r}\left(k_{1}-38\right)
$$

To study the negative effects of human physiological decline and positive effects of experience on worker performance a learning model [17] and aging model [18,19] combined. Hence, time to produce $n^{\text {th }}$ unit by Inexperience worker after the age of 38 years old is given as:

$$
\begin{aligned}
& T_{n}=[(100-(100-(0.57+0.012 \times K)(K-38) \times 0.01)] \times \\
& \left.\left.\left.\left[\left(Q+(1-Q) N^{C}\right) T_{\max }\right)\right]+\left[Q+(1-Q) N^{C}\right) T_{\max }\right)\right]
\end{aligned}
$$

And assembly time to produce $n^{\text {th }}$ unit by experience worker after the age of 38 years old is express as:

$$
\begin{aligned}
& T_{n}=[(100-(100-(0.57+0.012 \times K)(K-38) \times 0.01)] \times \\
& \left.\left.\left.\left[\left(Q+(1-Q) G^{C}\right) T_{\max }\right)\right]+\left[Q+(1-Q) G^{C}\right) T_{\max }\right)\right]
\end{aligned}
$$

\section{INCORPORATING HUMAN FACTORS INTO DES TOOL}

In a manual assembly flow line, material move sequentially through the line for successive operations until last task is completed. In a DES model using the Enterprise Dynamics package, human operators treated as assembler as the same function as servers. However, in reality, human acts as a much complex system with their abilities, which are subject to human and many other factors [2]. Few studies were reported in the literature relating to human factors being considered in DES applications [2, 9, 13]. In these studies, human factors can be manipulated externally by a series of MS Excel worksheet containing key input/output data in terms of cognitive and physical elements for DES models. Thus, the possible and realistic assignment of selected assembly tasks for each worker can be quantified [2, 9, 13].

For this study, a human factor decision tool called AutoHmot created and used as an aid for manufacturing system designers and analysists to examine system performance by considering effects of some human factors. With this tool, assembly time associated with worker aging and job experience can be integrated into a DES model providing simulation results of human performance at an early design stage of a manufacturing process. Figure 1 shows the AutoHmot graphical user interface developed using the java based JBuilder tool.

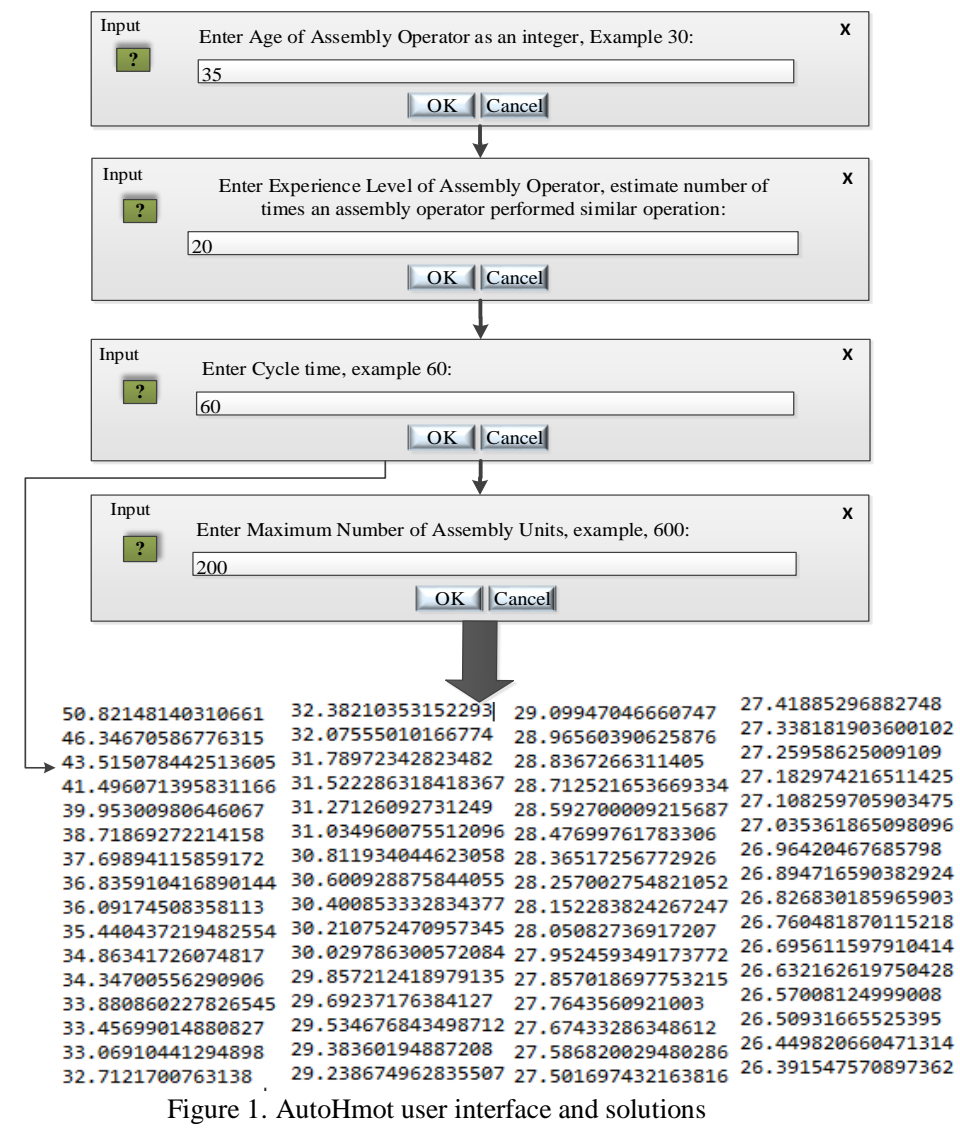

Figure 2 shows data obtained from AutoHmot, it indicates assembly time of a worker at full capaicty of 39 years old declines over the increasing number of assembled units this work completed.

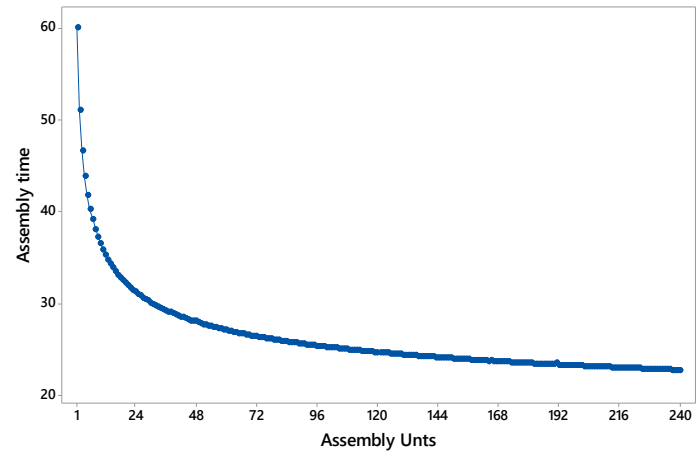

Figure 2. Assembly time vs assembly units completed by a worker at full capacity of 38 years old

Figure 3 illustrates mechanism of the integrated DES model interfaced with AutoHmot. Once the relevant 
information data are collected and entered into AutoHmot by users, assembly time will be computed. One of the benefits of this developed tool is that it requires minimum inputs from uses. a worker. In total, 240 units of product assembled. The literature study shows that individual performance of human workers usually approaches their full capacity at the age of

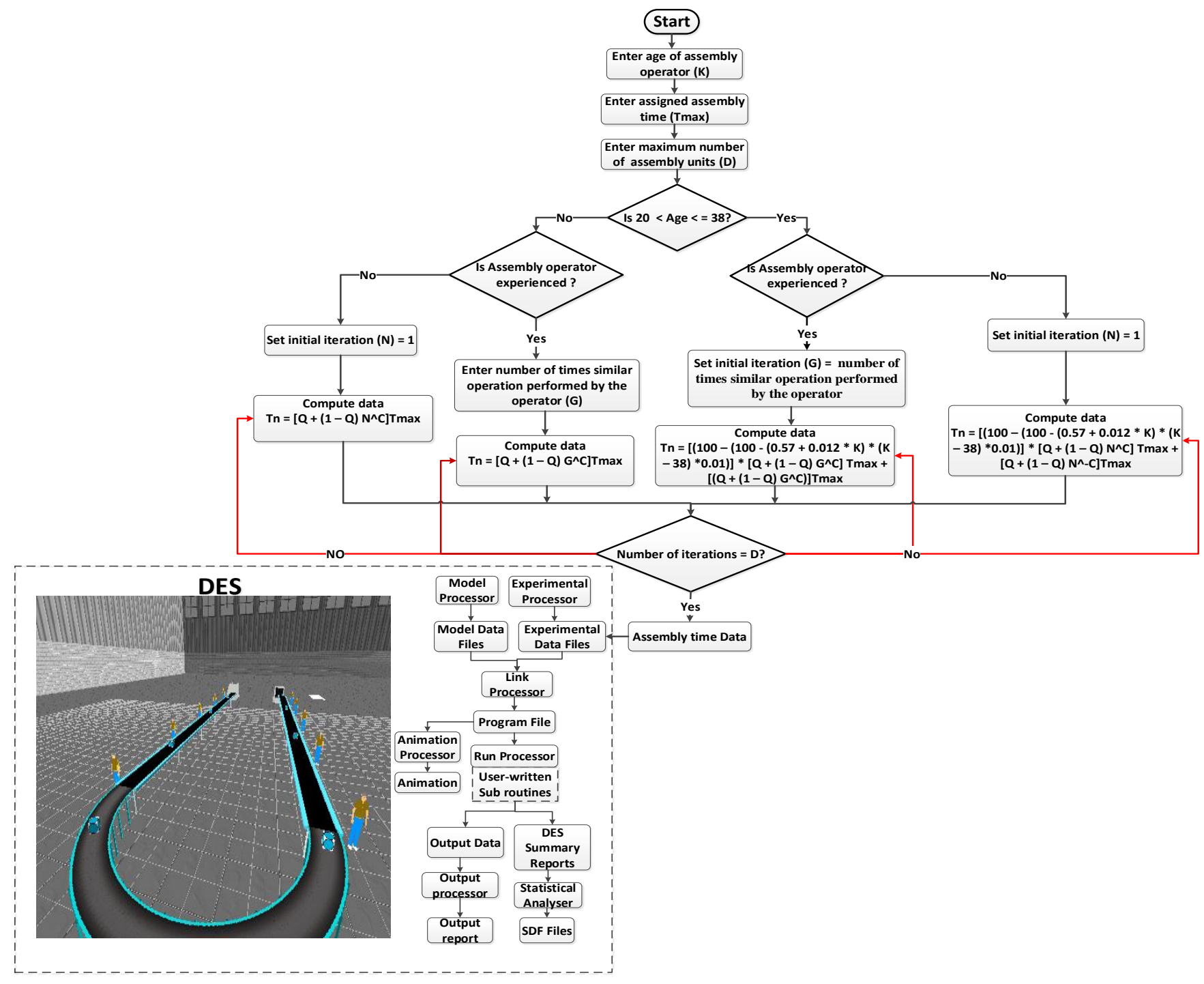

Figure 3. Integration of human elements into a DES model

The data information required from AutoHmot include:

1. Existing age of an assembly operator

2. Experience level of the tasks performed by assembly operator

3. The cycle time in which time is assigned to assemble a unit

4. The total number of assembly units that need to be completed

\section{SIMULATION RESULTS}

For this study, a DES model of a human centered assembly line constructed with each workstation manned by
38 years old. After this age, it may start to decline over the increasing age of workers [18]. Figure 4 shows the simulation result of assembly time of workers over the increase of age, it shows that assembly time for workers without prior experience increases by an average of $1 \%$ per year. While for the experienced workers, it decreases by an

average of $1.28 \%$, increase by $4.02 \%$ at the age of 40 years old and increased by $28.36 \%$ at the age of 70 years old. For highly experienced workers, assembly time decreased by an average of $3.18 \%$ at age of 40 years old, increased by $2.2 \%$ at the age of 45 years old, and increased by $27.1 \%$ at age of 70 years old. 


\section{$\square$ Inexperiece $\square$ Experienced $\square$ Highly experienced}

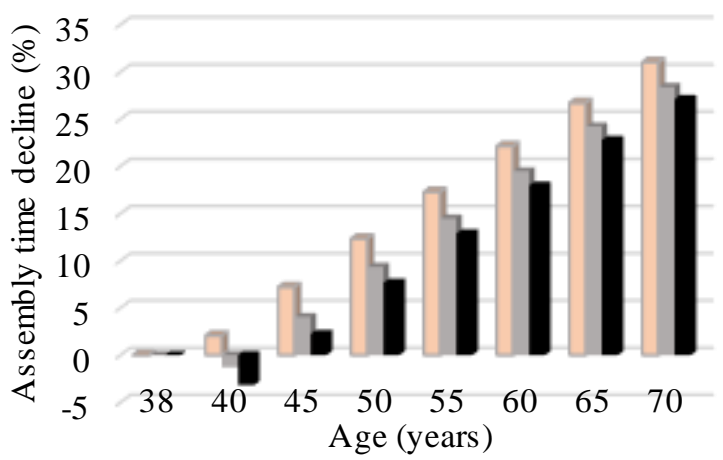

Figure 4. Decline rate (\%) of assembly time over the increase of age

\section{CONCLUSIONS}

The existing DES tools in the market do not sufficiently offer capabilities for system analysts to evaluate performance of manufacturing systems incorporating human factors. For a human centred assembly system, it may lead to a poor estimation in simulation results without considering effects of human factors [2]. The result from this study indicates that assembly time of workers increases over the increase of their age. Also, assembly time is affected by levels of experience of assembly worker. The paper presents a framework of a user-friendly integrated DES tool that allows manufacturing system designers to examine the overall performance of a human centred system with considerations of effects of human factors (age and experience).

\section{REFERENCES}

[1] Glock, C. H., Grosse, E. H., Kim, T., Neumann, W. P., \& Sobhani, A. (2019). An integrated cost and worker fatigue evaluation model of a packaging process. International Journal of Production Economics, Vol. 207, 107-124.

[2] Wang, Q., Sowden, M., \& Mileham, A. R. (2013). Modelling human performance withing an automotive engine assembly line. International Journal of Advanced Manufacturing Technology vol. 68(1-4), 141-148.

[3] Nof, S. Y., Wilhelm, W. E., \& Warnecke, H.-J. (1997). Industrial Assembly. London: Chapman \& Hall.

[4] Samy, S. N., \& Elmaraghy, H. (2010). A model for measuring product assembly complexity. Internationnal Journal l of Integrated Manufacturing, Vol. 23(11), 1015-1027.
[5] Greasley, A., \& Owen, C. (2018). Modelling people's behaviour using discrete-event simulation: A review. International Journal of Operations and Production Management, Vol. 38(5), 1228 - 1244

[6] Boenzi, F., Digiesi, S., Mummolo, G., \& Romano, V. A. (2015). Modelling workforce aging in job rotation problems. IFACPapersOnline, Vol. 48(3), 604-609.

[7] Lassila, A. M., Saad, S. M., \& Perera, T. (2005). Modelling and Simulation of Human - Centred Assembly Systems - A Real Case Study. In Emerging Solutions for Future Manufacturing System, 405412.

[8] Kolus, A., Wells, R., \& Neumann, P. (2018). Production quality and human factors engineering: A systematic review and theoretical framework. Applied Ergonomics, Vol. 73,55-89.

[9] Perez, J., Looze, M. d., Bosch, T., \& Neumann, W. (2014). Discrete event simulation as an ergonomic tool to predict workload exposures during systems design. International Journal of Industrial Ergonomics, Vol. 44, pp. 298 - 306.

[10] Digiesi, S., Kock, A. A., Mummolo, G., \& Rooda, J. E. (2009). The effect of dynamic worker behaviour on flowline performance. International Journal of Production Economics, Vol. 120(2), 368-377.

[11] Mason, S., Baines, T., John, K. M., \& Ladbrook, J. (2005). Improving The Design Process for Factories: Modelling Human Performance Variation. Journal of Manufacturing Systems, Vol. 24(1),44-54.

[12] Wang, Q., \& Chatwin, C. R. (2005). Key issues and developments in modelling and simulation-based technologies for manufacturing system analysis, design and performance evaluation. International Journal of Advanced Manufacturing Technology, Vol. 25, 1254-1265.

[13] Neumann, W. P., \& Medbo, P. (2017). Simulation operator learning during production ramp-up in parallel vs serial flow production. International of Production Research, Vol. 55(3), 845-857.

[14] Wang, Q., \& Abubakar, M. (2017). Human Factors and Their Effects on Human-Centred Assembly Systems - A Literature Review-Based Study. IOP Conf. Series: Materials Science and Engineering 239 (2017) 012006.

[15] Abubakar, M. I., \& Wang, Q. (2018a). Modeling and Simulation of Aging on Performance of Assembly Workers through a Learning Curve. International Journal of Modeling and Optimization, Vol 8(3), 183-187.

[16] Quinones, M. A., Ford, J. K., \& Teachout, M. S. (1995). Relationship between work experience and job performance: A conceptual and meta analytic review. Personal Psychology, Vol. 48, 887-910.

[17] Anzanello, M. J., \& Fogliatto, F. S. (2011). Learning curve models and applications: literature review and research directions. International Journal of Industrial Ergonomics, Vol. 41, 573-583.

[18] Abubakar, M. I., \& Qian, W. (2019). Key human Factors and their effects on human centered assembly systems. International Journal of Industrial Ergonomics, Vol. 69, 48-57.

[19] Abubakar, M. I., \& Wang, Q. (2018b). Incorporating Learning and Aging Attributes of Workers into A DES Model. International Conference on Robotics and Automation Sciences, (pp. 160-163). Wuyan: IEEE. 